\title{
Crecimiento económico y concentración del ingreso: sus efectos en la pobreza del Brasil
}

\author{
Jair Andrade Araujo, Emerson Marinho \\ y Guaracyane Lima Campêlo
}

\section{Resumen}

Para analizar los efectos del crecimiento económico y la desigualdad de los ingresos en la pobreza del Brasil se utilizan datos de panel para los estados brasileños de 1995 a 2009 y se evalúa la hipótesis de Bourguignon (2003) de que a mayor desigualdad en un país, menos eficaz resultará el crecimiento económico para reducir la pobreza. Estimamos las elasticidades pobreza-ingreso y pobrezadesigualdad mediante dos modelos econométricos dinámicos basados en el método generalizado de momentos de Arellano y Bond (1991), Arellano y Bover (1995) y Blundell y Bond (1998). Se concluye que a menor desarrollo inicial y mayor nivel de desigualdad inicial, menor incidencia tiene el aumento de los ingresos en la reducción de la pobreza. Las regiones con un nivel de desarrollo inicial limitado, una elevada desigualdad inicial, o ambos, presentan condiciones menos favorables para reducir la pobreza mediante el aumento del ingreso.

\section{Palabras clave}

Crecimiento económico, distribución del ingreso, pobreza, mitigación de la pobreza, modelos econométricos, Brasil

\section{Clasificación JEL}

D60, D63, C33

\section{Autores}

Jair Andrade Araujo es Profesor de la Maestría en Economía Rural y Jefe del Departamento de Economía Agrícola de la Universidad Federal de Ceará (Brasil). Correo electrónico: jairandrade@ufc.br.

Emerson Marinho es Profesor del Programa de Posgrado en Economía de la Universidad Federal de Ceará (Brasil). Correo electrónico: emarinho@ufc.br.

Guaracyane Lima Campêlo es Profesora del Departamento de Economía y Finanzas del campus de Sobral de la Universidad Federal de Ceará (Brasil). Correo electrónico: cyanelc@gmail.com. 



\section{Introducción}

Las políticas de desarrollo de muchos países están orientadas principalmente a mejorar el bienestar de la población. Entre los muchos objetivos que entraña esta meta, la reducción de la pobreza es fundamental, sobre todo si se toma en cuenta que la pobreza a menudo reviste un carácter persistente a pesar de la creciente capacidad de la economía de generar riqueza.

Según Rocha (2006), es evidente que el crecimiento económico no beneficia necesariamente a todos los miembros de una sociedad, por más que efectivamente se lo haya alcanzado. Obviamente, los países ricos se esfuerzan por erradicar la pobreza residual, al tiempo que en los países en desarrollo el crecimiento económico ha exacerbado las desigualdades sociales.

En años recientes se ha puesto en el centro de los debates la pregunta acerca de cómo los cambios en los niveles de ingreso y la desigualdad repercuten en la reducción de la pobreza, pues se ha comprobado que en muchos países el crecimiento económico aún tiene que resolver los problemas relacionados con la pobreza.

Por ejemplo, Cline (2004) analizó el caso de varios países durante la década de 1990 y concluyó que la pobreza efectivamente disminuyó en muchos de ellos como consecuencia del crecimiento económico. No obstante, algunos países que no tuvieron un crecimiento económico significativo también lograron reducir la pobreza. Esto demuestra que el crecimiento económico por sí solo no explica los cambios en los niveles de pobreza y que la desigualdad en materia de ingresos reviste importancia capital como factor de reducción de la pobreza.

Chen y Wang (2001) estudiaron la pobreza y la desigualdad en China durante la década de 1990. Tras descomponer los cambios en el nivel de pobreza causados por el crecimiento económico y las variaciones en la desigualdad, llegaron a la conclusión de que eran los ricos quienes más se beneficiaban del crecimiento económico, dado que el ingreso medio del 20\% más rico aumentaba más que el ingreso medio general. Esto pone de relieve la importancia que tiene la desigualdad de los ingresos como variable en reducción de la pobreza.

Barros, Henriques y Mendonça (2001) hallaron que en el Brasil la incidencia de la pobreza era mayor que en la mayoría de los países con ingresos per cápita similares. Concluyeron que la desigualdad en materia de ingresos era el motivo por el cual el crecimiento económico era una herramienta relativamente ineficaz para reducir la pobreza. En otras palabras, el efecto que el crecimiento económico ejerce en la reducción de la pobreza era más acotado en el Brasil que en otros países con el mismo nivel de ingreso.

Si algún cambio en el nivel de pobreza es consecuencia de la redistribución del ingreso o del crecimiento económico (o de ambos), debería determinarse la importancia que reviste cada uno de ellos. En estudios recientes se ha procurado determinar qué factores afectan las elasticidades pobreza-ingreso y pobreza-desigualdad.

Por ejemplo, Ravallion y Chen (1997) utilizaron una muestra de países en desarrollo para calcular la elasticidad pobreza-ingreso -medida por el número de personas con un ingreso por debajo de la línea de pobreza (un dólar por día) - y obtuvieron un valor de la elasticidad de -3. Este resultado significa que, por cada incremento del 1\% en el ingreso medio, la proporción de personas con ingresos inferiores a la línea de pobreza disminuye un 3\%. Sin embargo, algunos países han logrado disminuir la pobreza de los hogares sin alcanzar un crecimiento económico significativo.

Bourguignon (2003) estimó la elasticidad pobreza-ingreso de un conjunto de países usando una distribución log-normal a fin de realizar una aproximación a la distribución del ingreso. Demostró que cuanto mayor es el ingreso medio y menor su concentración, mayor será la elasticidad resultante. 
Marinho y Soares (2003) han reunido evidencia empírica en el caso del Brasil. Estos investigadores estimaron la elasticidad de la pobreza en función del ingreso medio para los estados brasileños de 1985 a 1999 y llegaron a la conclusión de que un ingreso medio más alto llevaba a una elasticidad absoluta más elevada y que una mayor concentración del ingreso llevaba a una menor elasticidad absoluta. Los valores más altos de elasticidad pobreza-ingreso se observaron en los estados de São Paulo y Río de Janeiro.

En otro estudio sobre el Brasil, Hoffman (2004) siguió una metodología diferente a la de Marinho y Soares (2003) para estimar estas mismas elasticidades. Halló que ambas estimaciones arrojaban variaciones estándar muy similares entre los distintos estados.

Salvato y Araujo Junior (2007) utilizaron datos de los municipios brasileños para estudiar la relación entre crecimiento, pobreza y desigualdad, para lo que midieron la elasticidad de la pobreza en función del crecimiento económico y los cambios en la desigualdad de los ingresos. También llevaron a cabo pruebas para determinar la existencia de un efecto de interacción no lineal entre el crecimiento y la desigualdad inicial, con el propósito de corroborar la hipótesis de que una mayor inequidad está asociada con una menor eficiencia del crecimiento en la mitigación de la pobreza. Hallaron que, entre las principales regiones, la sudeste presentaba la mayor elasticidad, mientras que São Paulo fue el estado que obtuvo los mejores resultados. También observaron una correlación negativa entre el módulo de elasticidad y la desigualdad inicial, lo que implica que ante una desigualdad inicial mayor, el crecimiento económico tendrá una menor capacidad de reducir la pobreza, lo que corroboró la hipótesis de Bourguignon (2003). Los resultados también parecen indicar la existencia de una correlación negativa entre la elasticidad de la redistribución y la desigualdad inicial.

No obstante, estos interrogantes no se han esclarecido por completo, dado que, según Barreto (2005), aún no se ha llegado a un consenso acerca de la relación entre pobreza, crecimiento y desigualdad. Por lo tanto, es de suma importancia determinar los efectos que cada uno de estos factores ejerce en la pobreza.

Medir estas elasticidades constituye un elemento fundamental de la planificación de las políticas de aumento y redistribución del ingreso, habida cuenta de que la reducción de la pobreza se ve afectada tanto por los cambios en el crecimiento económico como por la reducción de la desigualdad, tal como señala Cline (2004).

Con estos factores en mente, el propósito de este trabajo es analizar en qué medida el crecimiento económico y las variaciones en la desigualdad de los ingresos repercuten en los cambios en el nivel de pobreza en el Brasil. Puesto que el crecimiento por sí solo no puede explicar las alteraciones del nivel de pobreza, hemos tratado la desigualdad de los ingresos como uno de los factores que sí la explican, con miras a evaluar la hipótesis de que, cuanta más desigualdad hay en un país, menos efectivo será el crecimiento económico para reducir la pobreza (Bourguignon, 2003).

A fin de comprobar estos efectos, se estimó la elasticidad de la pobreza en función del ingreso y la desigualdad. Estas variables se estimaron mediante un modelo econométrico dinámico de datos de panel desarrollado por Arellano y Bond (1991), Arellano y Bover (1995), y Blundell y Bond (1998). En el panel, las unidades de análisis son los estados del Brasil durante el período que va de 1995 a 2009.

Este artículo se divide en seis secciones, además de esta introducción. En la sección II se pasa revista a la literatura brasileña e internacional sobre la relación triangular entre pobreza, crecimiento económico y desigualdad, y se brinda una breve historia de la desigualdad en el Brasil. En la sección III se proporcionan definiciones teóricas de las elasticidades pobreza-ingreso y pobreza-desigualdad. En la sección IV se examina la base de datos, mientras que en la sección $V$ se presentan el modelo econométrico y sus métodos de estimación. En la sección VI se efectúa un análisis de los resultados y, por último, en la sección VII se exponen las conclusiones de este análisis. 


\section{La relación triangular entre pobreza, crecimiento económico y desigualdad}

En esta sección se pasa revista a la literatura sobre la relación triangular entre pobreza, crecimiento económico y desigualdad de los ingresos. La interacción entre estas tres variables proporciona suficientes fundamentos para diagnosticar en qué medida el aumento del ingreso o la disminución de la desigualdad afecta la reducción de la pobreza.

Bourguignon (2003) describe con claridad lo que llama el triángulo pobreza-desigualdadcrecimiento y argumenta que estas tres variables interactúan entre sí. En su artículo, asume que la distribución del ingreso presenta una función log-normal y atribuye los cambios en los niveles de pobreza a dos causas diferentes: i) el efecto de crecimiento, que se produce cuando ocurre un cambio proporcional en los niveles de ingreso no necesariamente acompañado por un cambio en el ingreso relativo, y ii) el efecto de distribución, que tiene lugar cuando cambia la distribución del ingreso relativo.

Este enfoque permite demostrar que los cambios en los niveles de pobreza pueden resultar del crecimiento económico (representado por incrementos del ingreso medio) o de una disminución de la desigualdad de ingresos.

En el gráfico 1, las curvas reflejan las densidades de la distribución del ingreso, que se representan en el eje horizontal mediante una escala logarítmica. El desplazamiento de la distribución inicial a la distribución final atraviesa una etapa intermedia - la transposición horizontal de la distribución inicial a la curva (I)-. Este cambio representa un incremento proporcional de todos los ingresos de la población a causa del efecto de crecimiento.

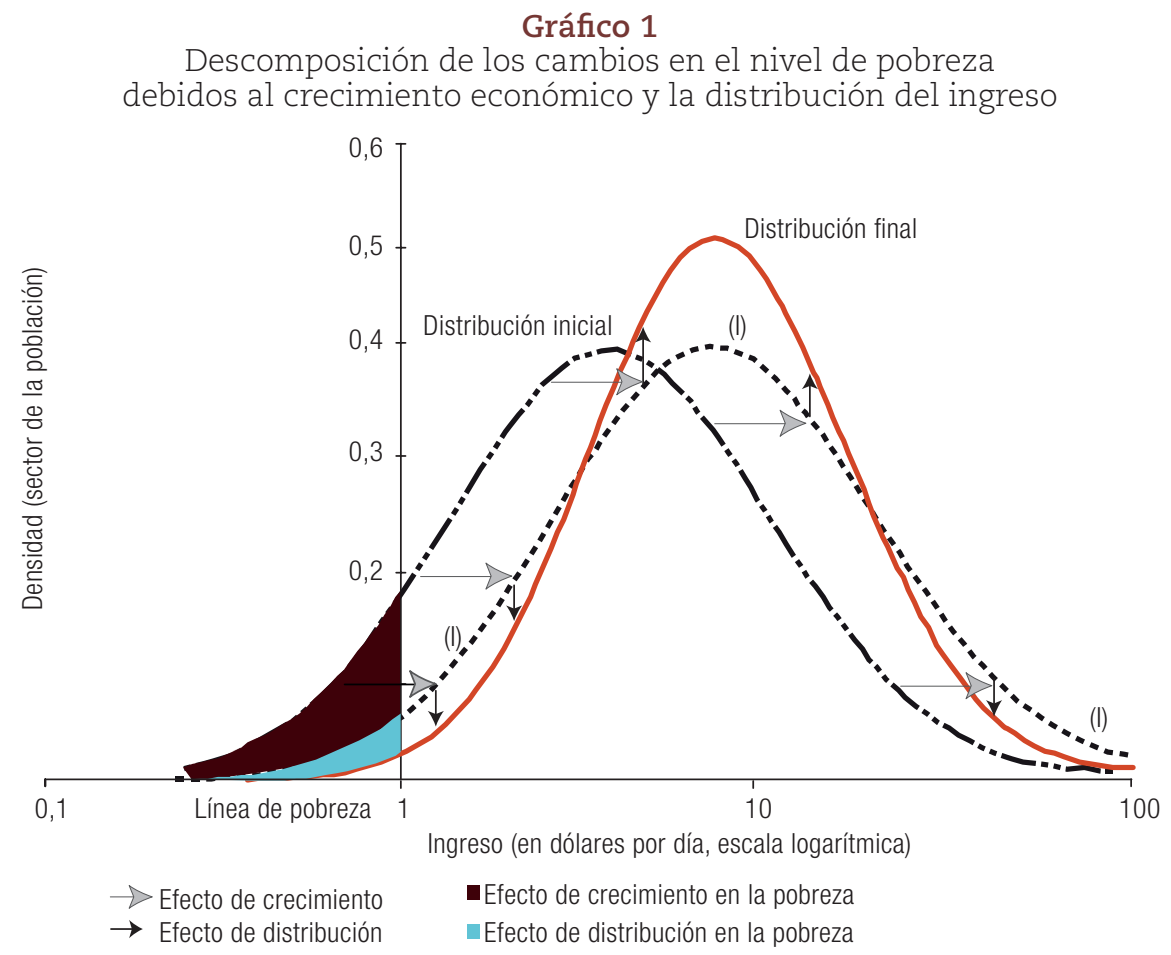

Fuente: F. Bourguignon, "The growth elasticity of poverty reduction: explaining heterogeneity across countries and time periods", Inequality and Growth: Theory and Policy Implications, T. Eicher y S. Turnovsky (eds.), Cambridge, Massachusetts, The MIT Press, 2003. 
Así, el cambio entraña un desplazamiento hacia la derecha de la densidad de la distribución del ingreso. Si se considera que $z$ representa la línea de pobreza, puede verse que se reduce el número de personas pobres. Esta disminución del porcentaje de personas pobres es exclusivamente producto del efecto de crecimiento.

El desplazamiento de la curva (I) hacia la distribución final ocurre con un ingreso medio constante y un cambio en la distribución del ingreso relativo - el efecto distributivo-. Por ende, desciende el nivel de pobreza sin que haya alteraciones en el ingreso medio de la población, porque disminuye la desigualdad en materia de ingresos. En otras palabras, una menor concentración del ingreso lleva a una reducción del número de personas con un ingreso por debajo de la línea de pobreza.

Bourguignon (2004) denomina a esta relación el "triángulo pobreza-desigualdad-crecimiento". A partir de ella, se han emprendido numerosos estudios para determinar y medir la relación entre los efectos del crecimiento y los cambios en los niveles de desigualdad en la reducción de la pobreza.

\section{La pobreza frente al crecimiento económico}

En varios estudios empíricos llevados a cabo en el Brasil y en el ámbito internacional se han analizado las interacciones entre el crecimiento económico y la pobreza. Entre los investigadores parece haber un amplio consenso acerca de que existen dos factores fundamentales para el estudio de la reducción de la pobreza: la tasa media de crecimiento y la desigualdad inicial en materia de ingresos.

Kraay (2004), por ejemplo, llevó a cabo una descomposición de la varianza de la pobreza para determinar la importancia del crecimiento económico a la hora de reducir la pobreza, para lo que utilizó una muestra de países en desarrollo que abarcó las décadas de 1980 y 1990. Su conclusión fue que los cambios en los índices de pobreza se debían al aumento del ingreso medio, de lo que se desprende que las políticas orientadas a promover el crecimiento económico resultaban esenciales para el bienestar de los pobres.

Es posible medir la relación entre crecimiento y reducción de la pobreza mediante las ecuaciones de elasticidad-ingreso y elasticidad-crecimiento. Si la elasticidad es alta, las políticas públicas para combatir la pobreza basadas en el crecimiento económico resultan más eficaces. En cambio, si la elasticidad es baja, las estrategias de reducción de la pobreza deberían incluir una combinación de crecimiento económico y algún tipo de redistribución del ingreso. Ravallion y Chen (1997) estimaron las elasticidades pobreza-ingreso y pobreza-desigualdad de 45 países. Los resultados correspondientes a los países con un bajo nivel de desigualdad mostraron que, si el ingreso aumentara un 1\%, la pobreza se reduciría un 4,3\%. Sin embargo, en países con una desigualdad elevada y el mismo nivel de crecimiento, la pobreza solo disminuiría el 0,6\%. La conclusión del estudio fue que el crecimiento por sí solo tenía pocos efectos en la pobreza, pero si la desigualdad tendía a disminuir a causa del crecimiento, el efecto en la pobreza era mucho más fuerte.

En otro estudio, Ravallion (2001) halló que la elasticidad pobreza-crecimiento era mucho más elevada en países que combinaban el crecimiento con algún tipo de política para reducir la desigualdad. Esto parece indicar que es preciso controlar por el componente de redistribución del ingreso la metodología usada para calcular la elasticidad pobreza-crecimiento. Por ejemplo, Ravallion (2005) estimó el crecimiento a favor de los pobres en China y la India en la década de 1990 y halló que los cambios en la distribución del ingreso eran desfavorables para los pobres, pues durante el período analizado la tasa de crecimiento de los ingresos de este sector de la población fue inferior a la tasa de crecimiento del ingreso de la población general. Este resultado arrojó una curva de incidencia del crecimiento con una pendiente positiva para los niveles de mayor ingreso. Sin embargo, el crecimiento de los ingresos a favor de los pobres era aún positivo, lo que indicaba un declive de la pobreza absoluta. 
Chen y Wang (2001) estudiaron la relación existente entre pobreza, ingresos y desigualdad en China durante la década de 1990 y llegaron a la conclusión de que, mientras que el crecimiento económico tendía a reducir la pobreza, la concentración del ingreso tendía a aumentarla. También hallaron que el incremento del ingreso medio resultaba más beneficioso para los ricos, pues solo el ingreso medio del $20 \%$ más rico aumentaba más que el ingreso medio de la población en general, lo que demostraba que la concentración del ingreso reduce el efecto positivo que el crecimiento económico tiene en los niveles de pobreza.

Stewart (2000) estimó que una tasa de crecimiento del PIB del 1,0\% llevaba a una merma de la pobreza del 0,21\% en Zambia, mientras que el mismo cambio redundaba en una disminución del 3,4\% en Malasia, discrepancia ocasionada por las diferencias que ambos países exhibían en materia de desigualdad de los ingresos.

Deininger y Squire (1996) confirmaron estas conclusiones y analizaron el efecto potencial que el crecimiento económico tenía en la reducción de la desigualdad en una muestra de países: hallaron que distintos niveles de desigualdad incidían de manera diferente sobre el crecimiento económico y que estos niveles guardaban una relación negativa con la pobreza.

Entre 1996 y 2000, Son (2004) determinó con una muestra de 84 países que el crecimiento económico contribuía a mitigar la pobreza en el 95\% de los casos. En los demás casos, la tasa de crecimiento era negativa o resultaba imposible extraer conclusiones debido a ambigüedades en el muestreo.

La literatura brasileña incluye algunos estudios sobre estos temas. Por ejemplo, Hoffmann (1995) observó una caída en los niveles de pobreza durante la década de 1970 junto con un crecimiento pronunciado del ingreso y una desigualdad relativamente invariable. En la década de 1980, la pobreza y la desigualdad aumentaron a causa de una inflación extremadamente alta.

En otro estudio, Hoffmann (2005) halló que un incremento del 1\% en el ingreso per cápita de los hogares del Brasil llevaba a un descenso del 0,84\% en la proporción de pobres y que el valor absoluto de esta elasticidad subía de la mano del ingreso y bajaba al reducirse la desigualdad.

Con datos correspondientes a 26 estados brasileños de 1985 a 1999, Marinho y Soares (2003) aplicaron un procedimiento metodológico que les permitió descomponer los cambios en los niveles de pobreza inducidos por modificaciones en el ingreso medio y la concentración del ingreso. Los resultados mostraron que la concentración del ingreso tenía una incidencia mayor que los niveles de ingreso en todos los estados del norte y que el crecimiento del ingreso revestía importancia estratégica para combatir la pobreza. En términos generales, su estudio mostró que con tasas medias más altas se obtenía una elasticidad absoluta más elevada, mientras que una mayor concentración del ingreso entrañaba una menor elasticidad absoluta.

Manso, Barreto y Tebaldi (2005) analizaron datos de la Encuesta Nacional de Hogares (PNAD) de 1995 a 2004 para hallar evidencia de las interacciones existentes entre el crecimiento de los ingresos, la reducción de la pobreza y el perfil de distribución de la riqueza. En su estudio, estos autores ampliaron el debate sobre el problema del desequilibrio regional del Brasil al evaluar el efecto del crecimiento económico en las tasas de pobreza. Este análisis les permitió aislar los efectos del crecimiento económico y la desigualdad de los ingresos para cada región del país. Los resultados parecen indicar que los componentes de la distribución del ingreso y el aumento del ingreso medio bastan para explicar la mayoría de las variaciones en los niveles de pobreza ocurridas en los diferentes estados brasileños. Hasta ahora, las conclusiones refuerzan la evidencia de que las políticas orientadas a combatir la pobreza mediante el crecimiento económico resultan más eficaces cuando van de la mano de una redistribución positiva del ingreso. 


\section{La pobreza frente a la desigualdad}

En esta subsección se analiza la relación entre pobreza y desigualdad que se aborda en la literatura. En general, muchos autores afirman que el porcentaje de pobres de una región determinada disminuye cuando se pone en marcha una política de crecimiento económico acompañada de una estrategia de redistribución del ingreso.

La desigualdad de los ingresos constituye un componente destacado del debate sobre la pobreza. La pobreza es un problema mundial que afecta a la sociedad moderna y que ha sido objeto de muchos estudios y que persiste a pesar del creciente nivel de riqueza material observado en todo el mundo. La cantidad de pobres de todo el planeta refleja el alcance y la gravedad que reviste la pobreza, que siempre va de la mano de la desigualdad.

Ravallion (2005) observó una relación no lineal entre la elasticidad pobreza-crecimiento y el nivel de desigualdad inicial en un conjunto de países subdesarrollados, por lo que planteó que el crecimiento económico resultaba poco beneficioso para los pobres si no servía también para disminuir la desigualdad. Se estima que un crecimiento del 1,0\% puede reducir la pobreza en hasta un 4,3\% en países con un bajo nivel de desigualdad. No obstante, en países que sufren una marcada desigualdad en materia de ingresos, el mismo crecimiento de un 1,0\% solo redunda en una disminución del 0,6\% en el porcentaje de pobres.

Por ende, uno de los factores que afectan las tasas de reducción de la pobreza dada una determinada tasa de crecimiento es el cambio en la distribución del ingreso. Esta fue también una de las conclusiones a las que arribaron Datt y Ravallion (2002) cuando midieron los cambios en el nivel de pobreza ocasionados por el efecto de la distribución del ingreso y el crecimiento. Los modelos diseñados para estimar la elasticidad de la pobreza deben incorporar la desigualdad de los ingresos como variable explicativa para evitar que los cambios en la distribución del ingreso se vean subsumidos en la elasticidad-crecimiento.

Según Bourguignon (2004), la reducción de la desigualdad de los ingresos es una herramienta útil para reducir la pobreza, y el crecimiento económico quizá no sea un elemento tan importante. Barros, Henriques y Mendonça (2001) hallaron resultados similares en el caso del Brasil. Estos autores hicieron hincapié en que la desigualdad en la distribución del ingreso era el motivo por el cual el crecimiento económico era menos efectivo de lo que podría ser para reducir la pobreza. En otras palabras, el efecto que el crecimiento tiene en la reducción la pobreza era más acotado en el Brasil que en otros países con el mismo nivel de ingreso.

Solo en unos pocos estudios se ha procurado explicar el nexo entre pobreza, crecimiento y desigualdad en el Brasil. Según Rocha (2006), aunque en el Brasil la pobreza haya persistido durante décadas, fue solo después de resolver el problema de la inflación cuando los problemas sociales comenzaron a recibir una atención prioritaria, y la reducción de la desigualdad es uno de los ejemplos. Esto puede explicar el limitado número de artículos donde se estudia el triángulo pobreza-crecimientodesigualdad en el Brasil.

Barreto (2005) considera que es posible reducir la pobreza en un plazo breve cuando un país en crecimiento logra una distribución del ingreso menos desigual. Por consiguiente, la puesta en marcha de políticas públicas destinadas a reducir la desigualdad, además de resolver este problema, también puede contribuir indirectamente a la consecución de otros objetivos de la política económica, como mayores tasas de crecimiento y la reducción de la pobreza. En general, en la literatura se sugiere que una baja significativa de las tasas de pobreza es fruto de un crecimiento económico combinado con políticas para reducir la desigualdad de los ingresos. El resultado conjunto de estos dos efectos es que la reducción de la pobreza representa un camino directo hacia la mejora del ingreso medio de los más pobres. 
Rocha (2006), por ejemplo, sostiene que en el Brasil la proporción de pobres decreció casi dos puntos porcentuales de 2001 a 2004. Según este autor, la reducción ocurrida en los primeros años de la década se debió a varios factores que tuvieron repercusiones regionales diferentes, entre ellos cambios distributivos en el rendimiento de la mano de obra y la ampliación de las prestaciones sociales.

Rocha (2006) plantea que en el Brasil la pobreza es muy persistente, debido, sobre todo, a la desigualdad, y que es posible reducirla mediante un crecimiento del ingreso y una mejor distribución. Sin embargo, existe consenso en que el énfasis debe ponerse en la reducción de la desigualdad de ingresos, pues si solo apunta a aumentarlos sin efectuar avances en materia de desigualdad, es posible que la meta de erradicar la pobreza se vea pospuesta a un futuro lejano.

En el cuadro 1 se presentan las tasas de reducción de la pobreza en el Brasil de 1995 a 2009. La proporción de personas pobres $\left(P_{0}\right)$ bajó del 38,70\% en 1995 al 23,50\% en 2009, cifra que, si bien es alta, representa una reducción de 15,20 puntos porcentuales.

\section{Cuadro 1}

Brasil: índices de pobreza, 1995-2009

(En porcentajes)

\begin{tabular}{llll}
\hline Año & $\mathrm{P}_{0}$ & Año & $\mathrm{P}_{0}$ \\
\hline 1995 & 38,7 & 2003 & 39,1 \\
\hline 1996 & 38,1 & 2004 & 37,0 \\
\hline 1997 & 38,5 & 2005 & 34,1 \\
\hline 1998 & 37,2 & 2006 & 29,6 \\
\hline 1999 & 39,0 & 2007 & 28,0 \\
\hline 2001 & 38,3 & 2008 & 25,4 \\
\hline 2002 & 38,2 & 2009 & 23,5 \\
\hline Diferencia -15,20 & & & \\
\hline
\end{tabular}

Fuente: Elaboración propia, sobre la base de datos de la Encuesta Nacional de Hogares (PNAD).

Así, al analizar este indicador puede notarse que en el período 1995-2009 en el Brasil se logró una reducción significativa de la pobreza.

\section{El crecimiento frente a la desigualdad}

En la literatura se ha analizado la relación entre el crecimiento económico y la desigualdad, tomando en consideración las causalidades que subyacen a estas dos variables. Muchas de estas cuestiones tienen que ver con la forma en que se genera la desigualdad y se reproduce en el tiempo y con la forma en que se interrelacionan la desigualdad y los procesos de desarrollo económico.

El punto de partida de esta teoría es la hipótesis de la U invertida de Kuznets (1955). Según esta hipótesis, la desigualdad primero debería aumentar al inicio del desarrollo económico, conforme la actividad económica pasa gradualmente de los sectores rurales a la industrialización (transferencia de mano de obra del sector menos productivo al más productivo). Posteriormente, una vez que la mayor parte de la fuerza de trabajo se encuentra ocupada en el sector industrial, la desigualdad debería disminuir.

Así, una política de desarrollo podría resumirse como la promoción del crecimiento económico de modo tal que también sirva para disminuir la desigualdad: con ingresos mayores y mejor distribuidos, debería quedar resuelto el problema de la pobreza.

Según Barreto (2005), en diversos estudios se ha analizado en qué medida la desigualdad repercute en el crecimiento económico. Distintos modelos muestran que la desigualdad puede tanto retardar como estimular el crecimiento. 
Por ejemplo, Alesina y Rodrik (1994) determinaron que la relación de causalidad entre crecimiento y desigualdad se basaba en tres factores: i) el gasto público y las políticas fiscales redistributivas deberían tener una relación negativa con el crecimiento debido a sus efectos perversos en la acumulación de capital; ii) las alícuotas tributarias deberían tender a ser proporcionales al ingreso, y los beneficios del gasto público deberían estar disponibles para todas las personas por igual, con la implicancia de que los niveles de gasto deberían ser inversamente proporcionales al ingreso, y iii) la carga fiscal impuesta por el gobierno debería ser la carga deseada por el votante medio, lo que entrañaría una menor acumulación de capital y, por ende, un menor crecimiento.

Stewart (2000) adopta un enfoque según el cual la desigualdad debería ser negativa para el crecimiento y plantea que una gran desigualdad: i) entraña inestabilidad política, incertidumbre, menos inversiones y un menor crecimiento; ii) conduce a una política populista de redistribución fiscal, desincentivos y menores tasas de crecimiento, y iii) afecta la conducta de los más ricos, que ejercen presión para recibir un trato impositivo preferencial, lo que induce a sobreinversiones en ciertas áreas y, por lo tanto, a un menor crecimiento.

Sin embargo, otros consideran que la desigualdad puede estimular el crecimiento económico. Bourguignon (1981) sostiene que la tendencia a ahorrar difiere entre ricos y pobres: es mayor entre los primeros, lo que implicaría que en las economías más inequitativas la tendencia a invertir sería más pronunciada y el crecimiento sería potencialmente más acelerado. Por el contrario, hay autores, como Barro (2000) y López (2004), que no hallan relación alguna entre la desigualdad y el crecimiento económico, y para quienes los niveles de inversión no tienen una dependencia significativa de la desigualdad. En el cuadro 2 se presentan algunos datos sobre la desigualdad de ingresos observada en el Brasil entre 1995 y 2009: el coeficiente de Gini, la relación entre el ingreso del 10\% más rico y el $40 \%$ más pobre, la relación entre el ingreso del $20 \%$ más rico y el $20 \%$ más pobre, la relación entre el ingreso del 10\% más rico y el 10\% más pobre, el ingreso medio per cápita de los hogares y el porcentaje de personas que se encuentran por debajo de la línea de pobreza.

Cuadro 2

Brasil: principales estadísticas relativas a la distribución del ingreso per cápita de los hogares, 1995-2009

\begin{tabular}{lcccccc}
\hline Año & $\begin{array}{c}\text { Coeficiente } \\
\text { de Gini }\end{array}$ & $\begin{array}{c}\text { Ingreso del 10\% más } \\
\text { rico como múltiplo } \\
\text { del 40\% más pobre }\end{array}$ & $\begin{array}{c}\text { Ingreso del 20\% más } \\
\text { rico como múltiplo } \\
\text { del 20\% más pobre }\end{array}$ & $\begin{array}{c}\text { Ingreso del 10\% más } \\
\text { rico como múltiplo } \\
\text { del 10\% más pobre }\end{array}$ & $\begin{array}{c}\text { Ingreso medio per } \\
\text { cápita de las familias } \\
\text { (en reales por mes) }\end{array}$ & $\begin{array}{c}\text { Personas por debajo } \\
\text { de la línea de } \\
\text { pobreza } \\
\text { (en porcentajes) }\end{array}$ \\
\hline 1995 & 0,601 & 23,7 & 27,4 & 67,0 & 520,6 & 19,7 \\
\hline 1996 & 0,602 & 24,2 & 29,3 & 74,9 & 529,7 & 19,5 \\
\hline 1997 & 0,602 & 24,2 & 28,7 & 72,3 & 529,0 & 19,8 \\
\hline 1998 & 0,601 & 23,6 & 27,5 & 67,2 & 534,5 & 19,1 \\
\hline 1999 & 0,595 & 22,7 & 26,2 & 63,2 & 504,4 & 19,9 \\
\hline 2001 & 0,597 & 22,9 & 26,9 & 68,4 & 511,9 & 19,7 \\
\hline 2002 & 0,590 & 21,9 & 24,7 & 59,2 & 511,9 & 19,5 \\
\hline 2003 & 0,585 & 21,1 & 24,3 & 59,4 & 481,9 & 20,1 \\
\hline 2004 & 0,575 & 19,5 & 22,0 & 51,7 & 497,9 & 18,9 \\
\hline 2005 & 0,572 & 19,2 & 21,3 & 49,7 & 528,4 & 17,5 \\
\hline 2006 & 0,560 & 18,3 & 20,4 & 47,5 & 577,5 & 15,2 \\
\hline 2007 & 0,550 & 17,7 & 20,2 & 49,0 & 592,5 & 14,4 \\
\hline 2008 & 0,540 & 16,8 & 18,9 & 44,0 & 622,6 & 12,9 \\
\hline 2009 & 0,540 & 16,3 & 18,6 & 43,8 & 637,4 & 12,2 \\
\hline
\end{tabular}

Fuente: Instituto de Estudios de Trabajo y Sociedad (IETS).

Nota: Se utilizó como parámetro la línea de pobreza correspondiente a 2009 -196,00 reales - deflactada por el índice nacional de precios al consumidor (INPC). 
En el cuadro 2 puede observarse que en el Brasil el ingreso del 10\% más rico equivalía a 23,7 veces el del 40\% más pobre en 1995, cifra que descendió a 16,3 veces en 2009. Las relaciones entre los ingresos del $10 \%$ más rico y el $10 \%$ más pobre, y entre el $20 \%$ más rico y el $20 \%$ más pobre también exhibieron un declive significativo durante este período, sobre todo en el caso de la primera, que cayó de 67,0 a 43,8. Mientras tanto, el ingreso familiar per cápita creció aproximadamente un 22,5\%. En la última columna se ve que la proporción de personas por debajo de la línea de pobreza se estabilizó en alrededor del 19\% y posteriormente se contrajo al 12\% en 2009.

Estos datos muestran que en años recientes la desigualdad de ingresos ha disminuido en el Brasil, lo que corrobora los resultados obtenidos por Neri (2006), Barros y otros (2007), y Hoffmann (2007). De manera similar, Manso, Barreto y Tebaldi (2005) observaron una reducción considerable de la desigualdad de los ingresos en el Brasil tras la puesta en marcha del Plan Real. De 1995 a 2004, el coeficiente de Gini exhibe una reducción del 2,71\%.

\section{La elasticidad de la pobreza en función de la desigualdad y el ingreso}

El propósito de determinar las elasticidades pobreza-ingreso y pobreza-desigualdad es analizar cómo repercuten en el nivel de pobreza tanto el crecimiento como los cambios en la desigualdad de los ingresos. Esta metodología fue propuesta originalmente por Bourguignon (2003).

El autor sigue la definición clásica propuesta por Foster, Greer y Thorbecke (1984), que mide la pobreza en función del porcentaje de personas pobres. Sobre la base de esta definición, la proporción de personas con un ingreso per cápita inferior a la línea de pobreza se obtiene con la siguiente fórmula:

$$
H_{t}=\operatorname{Pr}\left(y_{t}<z\right) \equiv F_{t}(z)
$$

donde la función $F_{t}(z)$ se obtiene a partir de la función de distribución del ingreso.

Por lo tanto, la proporción de la población con un ingreso por debajo de la línea de pobreza absoluta $z$ en el momento $t$ es igual a la probabilidad de que el ingreso $y_{t}$ sea inferior a la línea de pobreza. Así, la variación en el porcentaje de personas pobres entre dos períodos $t$ y $t^{\prime}$ se calcula con la siguiente fórmula:

$$
\Delta H=H_{t^{\prime}}-H_{t}=F_{t^{\prime}}(z)-F_{t}(z)
$$

Bourguignon (2003) asume que la curva de distribución del ingreso es log-normal y define el desplazamiento de la curva original que se muestra en el gráfico 1. Para calcular la curva de distribución final en función de las variaciones en el nivel de pobreza se utiliza la fórmula siguiente:

$$
\Delta H=H_{t^{\prime}}-H_{t} \approx\left[F_{t}\left(\frac{z}{\bar{y}_{t^{\prime}}}\right)-F_{t}\left(\frac{z}{\bar{y}_{t}}\right)\right]+\left[F_{t^{\prime}}\left(\frac{z}{\bar{y}_{t^{\prime}}}\right)-F_{t}\left(\frac{z}{\bar{y}_{t^{\prime}}}\right)\right]
$$

La primera expresión entre corchetes corresponde al efecto de crecimiento y la distribución del ingreso relativo $F_{t}$ se mantiene constante. La segunda expresión representa el efecto de la desigualdad, y se observa un cambio en la distribución del ingreso relativo, que permanece constante.

Así, las variaciones en el nivel de pobreza se ven influenciadas por dos efectos: el primero se debe al crecimiento del ingreso y el segundo es consecuencia de la desigualdad en su distribución. 
Según Epaulard (2003), el cambio relativo en el nivel de pobreza que resulta del aumento de los ingresos y el efecto de redistribución puede descomponerse de la siguiente manera:

$$
\frac{d H}{d t}=\frac{\partial H_{t}}{\partial \bar{y}_{t}} \frac{d \bar{y}_{t}}{d t}+\frac{\partial H_{t}}{\partial G_{t}} \frac{d G_{t}}{d t}
$$

En términos de elasticidad, tenemos:

$$
\frac{d H}{d t}=\varepsilon_{y}^{H} \frac{d \bar{y}_{t}}{d t} \frac{H_{t}}{\bar{y}_{t}}+\varepsilon_{G}^{H} \frac{H_{t}}{G_{t}} \frac{d G_{t}}{d t}
$$

donde el coeficiente de Gini se define como $G=2 \Phi\left(\frac{\sigma_{t}}{\sqrt{2}}\right)-1$. El término $\Phi(\cdot)$ es la función de densidad acumulada correspondiente a la distribución estándar normal (la distribución normal con una media de 0 y una desviación estándar igual a 1) y $\sigma_{t}$ representa la desviación estándar del logaritmo del ingreso. De este modo, Epaulard (2003) muestra que la elasticidad pobreza-ingreso $\varepsilon_{y}^{H}$ y la elasticidad pobreza-desigualdad $\varepsilon_{G}^{H}$ se definen con las siguientes expresiones:

$$
\begin{gathered}
\varepsilon_{\bar{y}}^{H}=\frac{\partial H_{t}}{\partial y_{t}} \frac{\bar{y}_{t}}{H_{t}} \equiv-\frac{1}{\sigma_{t}} \frac{\varnothing\left(\frac{\log \left(z / \bar{y}_{t}\right)}{\sigma_{t}}+\frac{1}{2} \sigma_{t}\right)}{\Phi\left(\frac{\log \left(z / \bar{y}_{t}\right)}{\sigma_{t}}+\frac{1}{2} \sigma_{t}\right)} \leq 0 \\
\varepsilon_{G}^{H}=\frac{\partial H_{t}}{\partial \sigma_{t}} \frac{\sigma_{t}}{H_{t}} \equiv \frac{1}{\sigma_{t}} \frac{\varnothing\left(\frac{\log \left(z / \bar{y}_{t}\right)}{\sigma_{t}}+\frac{1}{2} \sigma_{t}\right)}{\Phi\left(\frac{\log \left(z / \bar{y}_{t}\right)}{\sigma_{t}}+\frac{1}{2} \sigma_{t}\right)}\left(\frac{\log \left(z / \bar{y}_{t}\right)}{\sigma_{t}}+\frac{1}{2} \sigma_{t}\right) \gtreqless 0
\end{gathered}
$$

El autor también mostró que la elasticidad pobreza-ingreso $\left(\varepsilon_{\bar{y}}^{H}\right)$ y la elasticidad pobrezadesigualdad $\left(\varepsilon_{G}^{H}\right)$ disminuyen en términos absolutos en la línea de pobreza y el ingreso medio $\left(z / \bar{y}_{t}\right)$ y con la desviación estándar del logaritmo de ingresos $\left(\sigma_{t}\right)$. La elasticidad pobreza-ingreso siempre es positiva o nula. Por otro lado, la elasticidad pobreza-desigualdad puede estar por encima o por debajo de cero ${ }^{1}$.

Por consiguiente, el efecto que los cambios en la distribución del ingreso ejercen en la reducción de la pobreza es una función del crecimiento de los ingresos y el nivel de desigualdad. Ello significa que los cambios en el nivel de pobreza pueden ser el resultado tanto del crecimiento económico (representado por el aumento del ingreso medio) como de una menor desigualdad de los ingresos. No obstante, la reducción de la pobreza tiene efectos mucho más potentes cuando se combinan ambos factores.

\footnotetext{
1 Según Epaulard (2003), la elasticidad pobreza-desigualdad debería ser positiva a menos que el país tenga un ingreso medio muy bajo. Esta elasticidad será positiva siempre y cuando $\bar{y}_{t}<z \exp \left(-\frac{1}{2} \sigma_{t}^{2}\right)$.
} 


\section{La base de datos}

Los datos utilizados para realizar las estimaciones de los modelos econométricos presentados en la próxima sección se obtuvieron de la Encuesta Nacional de Hogares (PNAD) publicada por el Instituto Brasileño de Geografía y Estadística (IBGE). La muestra está compuesta por todos los estados brasileños y abarca de 1995 a 2009².

La variable correspondiente al ingreso per cápita de las familias se calcula dividiendo el ingreso familiar total por el número de miembros. A continuación, se determina el promedio aritmético de esta variable y se obtienen los valores correspondientes al ingreso medio de los estados incluidos en la muestra. Se espera observar una relación negativa entre la pobreza y esta variable. Cabe destacar que la economía brasileña experimentó un crecimiento del ingreso per cápita de 1995 a 2009.

En este artículo, se consideran pobres los hogares que viven con un ingreso familiar per cápita insuficiente para satisfacer sus necesidades básicas. Así, el indicador de pobreza absoluta utilizado es la proporción de personas pobres $\left(P_{0}\right)$. La línea de pobreza adoptada para elaborar este indicador fue la mitad del salario mínimo mensual ${ }^{3}$. El indicador de pobreza $P_{0}$ se define como $P_{0}=\frac{q}{n}$, donde $n$ es el número total de personas y $q$ representa el número de personas con un ingreso familiar per cápita inferior a la línea de pobreza.

El método usado para medir la desigualdad es el coeficiente de Gini, calculado sobre la base del ingreso per cápita de los hogares a partir de los datos de la PNAD. Este coeficiente a menudo se utiliza para expresar la desigualdad en materia de ingresos y puede relacionarse con la denominada curva de Lorenz, que se define sobre la base de los puntos obtenidos al analizar las participaciones de los ingresos en función de las participaciones de la población, en orden ascendente. Basados en esta curva, luego calculamos los coeficientes de Gini para cada uno de los estados de 1995 a 2009. Tal como se explicó en la sección precedente, la relación entre el coeficiente de Gini y la pobreza debe ser positiva. En otras palabras, cuanto mayor sea la desigualdad, mayor será la pobreza.

Cabe poner de relieve que todas las variables monetarias se ajustaron a valores reales de 2009 usando el índice nacional de precios al consumidor (INPC) elaborado con datos de 2009.

\section{El modelo econométrico}

La especificación econométrica del modelo se basa en la contribución del crecimiento económico y las variaciones en la distribución del ingreso a los cambios en el nivel de pobreza. Aceptamos como hipótesis que la actual tendencia de la pobreza apunta a autoperpetuarse, a afectar la conducta futura de la pobreza o a tener ambas consecuencias ${ }^{4}$. Para explorar estas hipótesis, se analizó la relación entre los cambios en la pobreza y sus determinantes por medio de un modelo de regresión dinámico de datos de panel basado en la siguiente definición $n^{5}$

$$
\Delta \ln \left[P_{0, i t}\right]=\beta_{0}+\beta_{1} \Delta \ln \left[P_{0, i t-1}\right]+\beta_{2} \Delta \ln \left[\bar{Y}_{i t}\right]+\beta_{3} \Delta \ln \left[\operatorname{Gini}_{i t}\right]+\eta_{t}+\mu_{i t}
$$

\footnotetext{
2 La PNAD no se llevó a cabo en 2000, por lo que se calcularon promedios aritméticos de las variables de 1999 a 2001 para llenar este vacío. En la muestra no se incluyeron los antiguos estados de la región norte, debido a la falta de datos correspondientes a las zonas rurales antes de 2004.

3 Esta línea también fue utilizada por Rocha (2006), Barreto (2005) y Marinho y Soares (2003). Sin embargo, la línea de cambio puede modificar el resultado.

4 Ribas, Machado y Golgher (2006) hallaron evidencia de la persistencia de la pobreza en el Brasil.

5 Puede consultarse este modelo en Bourguignon (2003), y en Kalwij y Verschoor (2004). Sin embargo, estos autores no creen que la pobreza pueda desarrollar una conducta dinámica.
} 
Las variables de este modelo se definen con las siguientes fórmulas:

$\Delta \ln P_{0, i t}=\ln P_{0 ; i t}-\ln P_{0 ; i t-1}$, que representa el cambio en la proporción de personas que son pobres entre dos períodos; $\Delta \ln \bar{Y}_{i t}=\ln \bar{Y}_{i t}-\ln \bar{Y}_{i t-1}$, que representa la variación en el ingreso medio per cápita de los hogares; y $\Delta \ln G i n i_{i t}=\ln G i n i_{i t}-\ln G i n i_{i t-1}$, que representa el cambio en la concentración del ingreso medido con el coeficiente de Gini $i$, mientras que $\eta_{i}$ representa los efectos aleatorios no observables de las personas y $\mu_{i t}$ representa las perturbaciones aleatorias. Las variables del modelo se definen mediante un logaritmo natural donde subíndice $i$ representa el estado y $t$ es el período de tiempo. Así, los parámetros $\beta_{2}$ y $\beta_{3}$ son la elasticidad pobreza-ingreso $\varepsilon_{y}^{P_{0}}$ y la elasticidad pobreza-desigualdad $\varepsilon_{G}^{P_{0}}$. Cabe tener presente que estas elasticidades no varían con el tiempo.

Una ampliación de este modelo efectuada por Kalwij y Verschoor (2004) permite que la elasticidadingreso y la elasticidad-desigualdad se modifiquen con el transcurso del tiempo dependiendo del nivel de desarrollo inicial inverso (la línea de pobreza dividida por el ingreso inicial per cápita de los hogares y el nivel de desigualdad inicial) ${ }^{6}$. Al introducir estas variables en el modelo, se tiene como objeto corroborar la hipótesis de que el crecimiento resulta menos eficaz como herramienta para luchar contra la pobreza cuando la desigualdad inicial es mayor (hipótesis de Bourguignon). Este modelo dinámico se puede describir con la siguiente fórmula:

$$
\begin{gathered}
\Delta \ln \left[P_{0, i t}\right]=\beta_{0}+\beta_{1} \Delta \ln \left[P_{0, i t-1}\right]+\beta_{2} \Delta \ln \left[\bar{y}_{i t}\right]+\beta_{3} \Delta \ln [\bar{y}] \ln \left[G_{i 0}\right]+\beta_{4} \Delta \ln \left[\bar{y}_{i t}\right] \ln \left[\frac{z_{i t}}{y_{i 0}}\right] \\
\beta_{5} \Delta \ln \left[\operatorname{Gini}_{i t}\right]+\beta_{6} \Delta \ln \left[\operatorname{Gini}_{i t}\right] \ln \left[G i n i_{i 0}\right]+\beta_{7} \Delta \ln \left[G i n i_{i t}\right] \ln \left[\frac{z_{i t}}{\bar{y}_{i 0}}\right]+\beta_{8} \ln \left[G_{i 0}\right] \\
\beta_{9} \ln \left[\frac{z_{i t}}{\bar{y}_{i 0}}\right]+\eta_{i}+\mu_{i t}
\end{gathered}
$$

donde, además de las variables $\Delta \ln \left[P_{i t}\right], \Delta \ln \left[\bar{y}_{i t}\right]$ y $\Delta \ln \left[G i n i_{i t}\right]$, que siguen las formulaciones presentadas anteriormente, tenemos $\Delta \ln \left[\bar{y}_{i t}\right] \ln \left[G_{i 0}\right]$ y $\Delta \ln \left[\bar{y}_{i t}\right] \ln \left[\frac{z_{i t}}{y_{i 0}}\right]$, que representan las interacciones entre el ingreso medio per cápita de los hogares y el coeficiente de Gini inicial en el estado $i\left(G_{i 0}\right)$ y el nivel de desarrollo inicial inverso $\frac{z_{i t}}{y_{i 0}}$ (la línea de pobreza dividida por el ingreso inicial per cápita de los hogares). De igual modo, las variables $\Delta \ln \left[\operatorname{Gini}_{i t}\right] \ln \left[G_{i 0}\right]$ y $\Delta \ln \left[\operatorname{Gini}_{i t}\right] \ln \left[\frac{z_{i t}}{\bar{y}_{i 0}}\right]$ representan las interacciones entre el índice de desigualdad de Gini y el índice de desigualdad inicial asociado con el estado $i$ y el nivel de desarrollo inicial inverso.

Las hipótesis adoptadas en estos modelos son $E\left[\eta_{i}\right]=E\left[\mu_{i t}\right]=E\left[\eta_{i} \mu_{i t}\right]=0$ y $E\left[\mu_{i t} \mu_{i s}\right]=0$ para $i=1.2, \ldots \ldots, N$ e $\forall t \neq s$. Además, hay una hipótesis estándar relativa a las condiciones iniciales: $\Delta \ln P_{i t}$ : $E\left[\Delta \ln P_{i t-1} \mu_{i t}\right]=0$ para $i=1.2, \ldots, N$ y $t=1.2, \ldots ., T$ (Ahn y Schmidt, 1995).

Por lo tanto, la segunda especificación del modelo toma en cuenta que las elasticidades de la pobreza en función del ingreso familiar medio per cápita y la desigualdad dependen de la desigualdad inicial y de la relación entre la línea de pobreza y el ingreso inicial medio per cápita de los hogares.

Naturalmente, los coeficientes $\beta_{2}$ y $\beta_{5}$ no se interpretan como elasticidad-ingreso y elasticidaddesigualdad. Para calcular estas elasticidades, es necesario tomar en cuenta los términos de interacción. Así, las elasticidades pobreza-ingreso y pobreza-desigualdad ahora se definen, respectivamente, como:

$$
\varepsilon_{\bar{y}_{i t}}^{P_{0}}=\beta_{2}+\beta_{3} \ln \left[G_{i 0}\right]+\beta_{4} \ln \left[\frac{z_{i t}}{\bar{y}_{i 0}}\right]
$$

\footnotetext{
${ }^{6}$ De manera similar, Kalwij y Verschoor (2004) creen que la pobreza no exhibe una conducta dinámica con el tiempo.
} 


$$
\varepsilon_{G_{i t}}^{P_{0}}=\beta_{5}+\beta_{6} \ln \left[G_{i 0}\right]+\beta_{7} \ln \left[\frac{z_{i t}}{\bar{y}_{i 0}}\right]
$$

Puede observarse que las elasticidades pobreza-ingreso y pobreza-desigualdad efectivamente varían con el tiempo.

Las técnicas tradicionales de estimación resultan inadecuadas para los dos modelos expuestos, sobre todo por dos problemas econométricos. El primero es la presencia de efectos inobservables de los individuos $\eta_{i}$, junto con la variable dependiente retrasada $\Delta \ln P_{k, i t-1}$, que aparece del lado derecho de la ecuación. En este caso, la omisión de los efectos fijos individuales en el modelo de panel dinámico hace que los estimadores de mínimos cuadrados ordinarios (MCO) resulten sesgados e inconsistentes.

Por ejemplo, la correlación positiva demostrable entre la variable dependiente retrasada y los efectos fijos significa que el estimador del coeficiente $\beta_{1}$ presenta un sesgo ascendente. Por otra parte, el estimador intragrupos (que corrige por la presencia de efectos fijos) genera una estimación de $\beta_{1}$ con un sesgo descendente en paneles tcon una dimensión temporal acotada (Judson y Owen, 1999).

Con miras a subsanar estos problemas, Arellano y Bond (1991) propusieron un estimador diferenciado por el método generalizado de momentos (MGM). Dicho método consiste en eliminar los efectos fijos mediante la ecuación de primera diferencia. En consecuencia, para ambos modelos se obtienen las siguientes fórmulas:

$$
\begin{gathered}
\Delta\left[\Delta \ln \left[P_{0, i t}\right]\right]=\beta_{1} \Delta\left[\Delta \ln \left[P_{0, i t-1}\right]\right]+\beta_{2} \Delta\left[\Delta \ln \left[\bar{Y}_{i t}\right]\right]+\beta_{3} \Delta \Delta\left[\ln \left[\operatorname{Gini}_{i t}\right]\right]+\Delta \mu_{i t} \\
\Delta\left[\Delta \ln \left[P_{0, i t}\right]\right]=\beta_{0}+\beta_{1} \Delta\left[\Delta \ln \left[P_{0, i t-1}\right]\right]+\beta_{2} \Delta\left[\Delta \ln \left[\bar{Y}_{i t}\right]\right]+\beta_{3} \Delta\left[\Delta \ln [\bar{Y}]\left[G_{i 0}\right]\right] \\
+\beta_{4} \Delta\left[\Delta \ln \left[\bar{y}_{i t}\right] \ln \left[\frac{z_{i t}}{\bar{y}_{i 0}}\right]\right]+\beta_{5} \Delta\left[\Delta \ln \left[\operatorname{Gini}_{i t}\right]\right]+\beta_{6} \Delta\left[\Delta \ln [G \operatorname{Gini} i t] \ln \left[G_{i 0}\right]\right] \\
+\beta_{7} \Delta\left[\Delta \ln \left[\operatorname{Gini}_{i t}\right] \ln \left[\frac{z_{i t}}{\bar{y}_{i 0}}\right]\right]+\beta_{8} \Delta\left[\Delta \ln \left[G_{i 0}\right]\right]+\beta_{9} \Delta\left[\ln \left[\frac{z_{i t}}{\bar{y}_{i 0}}\right]\right]+\Delta \mu_{i t}
\end{gathered}
$$

donde para una variable $w_{i t}$, toda $\Delta \ln \left[w_{i t}\right]=\ln \left[w_{i t}\right]-\ln \left[w_{i t-1}\right]$. En las ecuaciones (12) y (13), se correlacionaron $\Delta\left[\Delta \ln \left[P_{0, i t-1}\right]\right]$ y $\Delta \mu_{i t}$, por lo que los estimadores de MCO para sus coeficientes resultarán sesgados e incongruentes. Por lo tanto, es preciso utilizar variables instrumentales para $\Delta\left[\Delta \ln \left[P_{0, i t-1}\right]\right]$ en este caso. El conjunto de hipótesis adoptadas para las ecuaciones (8) y (9) implica que las condiciones de los momentos $E\left[\Delta\left[\Delta \ln P_{0, i t-S}\right] \Delta \mu_{i t}\right]=0$ para $t=3.4, \ldots . T$ y $s \geq 2$ son válidas. Sobre la base de estos momentos, Arellano y Bond (1991) proponen utilizar $\Delta \ln \left[P_{0, i t-s}\right]$ para $t=3.4, \ldots . T$ y $s \geq 2$ como instrumentos para las ecuaciones (12) y (13).

Respecto de las demás variables explicativas, existen tres situaciones posibles. Una variable explicativa $x_{i t}$ puede considerarse: i) estrictamente exógena si no está correlacionada con los términos de error pasados, presentes o futuros; ii) francamente exógena si solo está correlacionada con términos de error pasados, y iii) endógena si está correlacionada con los términos de error pasados, presentes o futuros. En el segundo caso, los valores retrasados de $x_{i t}$ correspondientes a uno o más períodos son instrumentos válidos para estimar las ecuaciones (12) y (13). En el último caso, los valores retrasados de $x_{i t}$ correspondientes a uno o más períodos son instrumentos válidos para las estimaciones de estas mismas ecuaciones. 
Por otra parte, Arellano y Bover (1995) y Blundell y Bond (1998) afirman que estos instrumentos resultan débiles cuando las variables explicativas y dependientes exhiben una persistencia marcada o cuando aumenta la varianza relativa de los efectos fijos, o en ambos casos. Ello permite obtener un estimador diferenciado por el método generalizado de momentos (MGM) que resulta incongruente y sesgado para los paneles $T$ pequeños.

Por ende, estos autores proponen una estimación basada en un sistema que combine las ecuaciones de nivel (8) y (9) y las ecuaciones de diferencia (12) y (13) a fin de reducir el sesgo y la falta de precisión. De aquí proviene el método generalizado de momentos (sistema MGM). En el caso de las ecuaciones de diferencia, el conjunto de instrumentos es el mismo que se describió anteriormente. En cuanto a una regresión del nivel, los instrumentos apropiados son las diferencias retrasadas de las variables respectivas. Por ejemplo, si se asume que las diferencias en las variables explicativas no están correlacionadas con los efectos fijos individuales para $t=3.4, \ldots . T$ y $E\left[\Delta\left[\Delta \ln P_{0, i 2}\right] \eta_{i}\right]=0$ para $i=1.2 .3, \ldots, N$, entonces las diferentes variables explicativas y $\left.\Delta\left[\Delta \ln P_{k, i t-1}\right] \eta_{i}\right]$, si son exógenas o francamente exógenas, aún serán instrumentos válidos para las ecuaciones de nivel. Lo mismo ocurre si son endógenas, pero con instrumentos que son las variables explicativas en la diferencia retrasada de un período y $\Delta\left[\Delta \ln P_{k, i t-1}\right]$.

Por último y como medio para comprobar la solidez y congruencia del modelo, Arellano y Bond (1991) proponen dos tipos de pruebas diferentes: las pruebas de Hansen y Sargan, que permiten determinar si los instrumentos utilizados y los instrumentos extra requeridos por el MGM son válidos. Finalmente, las pruebas estadísticas de Arellano y Bond corroboran si el error $\mu_{i t}$ tiene una correlación serial de primer orden y si $\Delta \mu_{i t}$ exhibe una correlación de segundo orden. En cuanto a la congruencia del estimador, se espera que $\mu_{i t}$ dé lugar a una correlación de primer orden, mientras que la serie $\Delta \mu_{i t}$ no debería presentar una correlación de segundo orden.

Cabe poner de relieve que el sistema MGM presentado en la sección siguiente se desprende de la estimación llevada a cabo con el estimador corregido con el método de Windmeijer (2005), a fin de prevenir que el estimador de varianza subestime las verdaderas varianzas de una muestra finita. Arellano y Bond (1991) propusieron la aplicación de este estimador en dos etapas. En la primera, se presupone que los términos de error son independientes y homocedásticos en el tiempo en sus estados respectivos. En la segunda etapa, los residuales obtenidos en la primera etapa se utilizan para efectuar una estimación coherente de una matriz de varianza y covarianza, lo que otorga laxitud al criterio de independencia y la hipótesis de homocedasticidad. El estimador de la segunda etapa es asintóticamente más eficiente que el de la primera.

\section{Resultados del modelo econométrico}

En esta sección se presentan los resultados de las estimaciones de los parámetros de los dos modelos, que se usarán para calcular las elasticidades pobreza-ingreso y pobreza-desigualdad.

Los resultados estimados para el primer modelo mediante los métodos de los mínimos cuadrados ordinarios, intragrupos y el sistema MGM pueden consultarse en el cuadro 3.

En el cuadro 3, el valor del coeficiente estimado para la variable $\Delta \ln \left[P_{i t-1}\right]$ aplicando el método generalizado de momentos (MGM) (columna [c]) se encuentra dentro de los valores de los coeficientes estimados para esta misma variable utilizando el método de los mínimos cuadrados ordinarios y el método intragrupos (columnas [a] y [b]). Así, el sistema MGM reduce el problema del sesgo de estimación, pues hay una variable dependiente retrasada un período del lado derecho de la ecuación (8). Nótese que en la columna [c] la significancia estadística del coeficiente estimado de $\Delta \ln \left[P_{0, i t-1}\right]$ confirma la hipótesis inicial de que la variación de la pobreza es persistente. 
Cuadro 3

Resultados de los modelos de regresión para $\Delta \ln \left[P_{0, i t}\right]$ : modelo 1

\begin{tabular}{|c|c|c|c|c|c|c|}
\hline & \multicolumn{2}{|c|}{$\begin{array}{l}\text { Mínimos cuadrados ordinarios } \\
\qquad[\mathrm{a}]\end{array}$} & \multicolumn{2}{|c|}{$\begin{array}{l}\text { Intragrupos } \\
{[\mathrm{b}]}\end{array}$} & \multicolumn{2}{|c|}{$\begin{array}{l}\text { Método generalizado } \\
\text { de momentos } \\
\text { [c] }\end{array}$} \\
\hline & Coeficiente & Valor $p$ & Coeficiente & Valor $p$ & Coeficiente & Valor $p$ \\
\hline$\Delta \ln \left[P_{0, i t-1}\right]$ & $\begin{array}{l}0,1840 \\
(0,0672)\end{array}$ & 0,00 & $\begin{array}{c}0,1529 \\
(0,0686)\end{array}$ & 0,02 & $\begin{array}{c}0,1139 \\
(0,0239)\end{array}$ & 0,00 \\
\hline$\Delta \ln \left[\bar{y}_{i t}\right]$ & $\begin{array}{l}-0,7654 \\
(0,0651)\end{array}$ & 0,00 & $\begin{array}{l}-0,7886 \\
(0,0658)\end{array}$ & 0,00 & $\begin{array}{l}-0,6899 \\
(0,0507)\end{array}$ & 0,00 \\
\hline$\Delta \ln \left[\right.$ Gini $\left._{i t}\right]$ & $\begin{array}{l}0,8785 \\
(0,1451)\end{array}$ & 0,00 & $\begin{array}{l}0,9046 \\
(0,1464)\end{array}$ & 0,00 & $\begin{array}{c}0,7799 \\
(0,1385)\end{array}$ & 0,02 \\
\hline \multirow[t]{2}{*}{ Constante } & $\begin{array}{l}-0,0079 \\
(0,0049)\end{array}$ & 0,11 & $\begin{array}{l}-0,0080 \\
(0,0050)\end{array}$ & 0,10 & $\begin{array}{l}-0,0114 \\
(0,0007)\end{array}$ & 0,00 \\
\hline & \multicolumn{2}{|c|}{$\begin{array}{l}F(3,269)=53,11 \\
\text { Prob }>F=0,0000 \\
R^{2}=0,37\end{array}$} & \multicolumn{2}{|c|}{$\begin{array}{l}F(3,249)=53,21 \\
\text { Prob }>F=0,0000\end{array}$} & \multicolumn{2}{|c|}{$\begin{array}{l}F(2,20)=124,30 \\
\text { Prob }>F=0,0000\end{array}$} \\
\hline \multicolumn{3}{|c|}{$\begin{array}{l}\mathrm{H}_{0}: \text { Ausencia de autocorrelación en los } \\
\text { residuales de primer orden }\end{array}$} & \multicolumn{2}{|c|}{ Valor $p$} & \multicolumn{2}{|c|}{0,001} \\
\hline \multicolumn{3}{|c|}{$\begin{array}{l}\mathrm{H}_{0} \text { : Ausencia de autocorrelación en los } \\
\text { residuales de segundo orden }\end{array}$} & \multicolumn{2}{|c|}{ Valor $p$} & \multicolumn{2}{|c|}{0,101} \\
\hline \multicolumn{3}{|c|}{ Prueba de Hansen } & \multicolumn{2}{|c|}{ Prob $>$ chi $^{2}$} & \multicolumn{2}{|c|}{0,288} \\
\hline \multicolumn{3}{|c|}{ Prueba de Sargan } & \multicolumn{2}{|c|}{ Prob $>$ chi $^{2}$} & \multicolumn{2}{|c|}{0,262} \\
\hline
\end{tabular}

Fuente: Elaboración propia.

Nota: i) Los valores entre paréntesis son las desviaciones estándar corregidas con el método de Windmeijer (2005); ii) los valores de la prueba de Hansen son los valores p de la hipótesis nula de que los instrumentos son válidos; iii) los valores de la prueba de Sargan son los valores p correspondientes a la validez de los instrumentos adicionales requeridos por el sistema MGM, donde se toman las variables explicativas en diferencias retrasadas como instrumentos para el sistema MGM y se retrasa $\Delta \ln \left[P_{0, i t-1}\right]$ y $\Delta \ln \left[\bar{y}_{i t}\right]$ un período, y iv) se dispone de 273 observaciones, 21 grupos y 17 instrumentos.

Los resultados estimados para los parámetros de la elasticidad-ingreso y la elasticidaddesigualdad fueron -0,69 y 0,78, respectivamente, tal como se aprecia en la columna [c]. Así, un aumento del 1,0\% en el ingreso per cápita redunda en una merma del 0,68\% en el porcentaje de pobres. Un incremento del 1,0\% en el índice de desigualdad conlleva una suba del 0,78\% en los niveles de pobreza. Cabe recalcar que los valores estimados para las elasticidades concuerdan con los valores de las elasticidades teóricas presentados en la sección III. También permiten corroborar los resultados obtenidos en estudios llevados a cabo en el ámbito internacional, como los de Kalwij y Verschoor (2004) y Bourguignon (2004), así como en el Brasil, como los de Marinho y Soares (2003), y Hoffmann (2004). Esto indica que las políticas orientadas a reducir la desigualdad son más eficaces para combatir la pobreza que aquellas solo destinadas a mejorar el ingreso medio.

En el cuadro 4 se presentan los resultados estimados para los parámetros de la ecuación (9). Una vez más, el valor del parámetro estimado para la variable $\Delta \ln \left[P_{i t-1}\right]$ está dentro del rango de los valores estimados para esta misma variable con el método de los mínimos cuadrados ordinarios (MCO) y con el método intragrupos (columnas [a] y [b]). Cuando en la estimación se utiliza el MGM, este parámetro no resulta estadísticamente significativo.

Entre los factores aislados que contribuyen de manera significativa al aumento de la pobreza, pueden mencionarse los siguientes en orden de importancia ascendente: la interacción entre los cambios en los ingresos y el nivel de desarrollo inicial inverso, la interacción entre los cambios en los ingresos y la desigualdad inicial en materia de ingresos, y la actual desigualdad de los ingresos. En la columna [c] del cuadro 4 se presentan valores positivos y significativos para estas variables.

El término de interacción entre los cambios en los ingresos y el nivel de desarrollo inicial inverso arroja un coeficiente estimado positivo y estadísticamente significativo, lo que también ocurre con el coeficiente de interacción entre los cambios en los ingresos y el nivel de desigualdad inicial, tal como reflejan los valores de la columna [c]. 
Cuadro 4

Resultados de los modelos de regresión para $\Delta \ln \left[P_{0, i t}\right]$ : modelo 2

\begin{tabular}{|c|c|c|c|c|c|c|}
\hline & \multicolumn{2}{|c|}{$\begin{array}{l}\text { Mínimos cuadrados ordinarios } \\
\qquad[\mathrm{a}]\end{array}$} & \multicolumn{2}{|c|}{$\begin{array}{l}\text { Intragrupos } \\
{[\mathrm{b}]}\end{array}$} & \multicolumn{2}{|c|}{$\begin{array}{l}\text { Método generalizado } \\
\text { de momentos } \\
{[\mathrm{c}]}\end{array}$} \\
\hline & Coeficiente & Valor $p$ & Coeficiente & Valor $p$ & Coeficiente & Valor $p$ \\
\hline$\Delta \ln P_{0, i t-1}$ & $\begin{array}{c}0,1463 \\
(0,0676)\end{array}$ & 0,03 & $\begin{array}{l}0,0425 \\
(0,0720)\end{array}$ & 0,55 & $\begin{array}{l}0,1301 \\
(0,0711)\end{array}$ & 0,08 \\
\hline$\Delta \ln \left[\bar{Y}_{i t}\right]$ & $\begin{array}{l}-0,3675 \\
(0,1485)\end{array}$ & 0,01 & $\begin{array}{l}-0,4137 \\
(0,1516)\end{array}$ & 0,00 & $\begin{array}{l}-1,0806 \\
(0,2936)\end{array}$ & 0,00 \\
\hline$\Delta \ln \left[\bar{Y}_{i t}\right] \ln \left[G_{i 0}\right]$ & $\begin{array}{c}0,4371 \\
(0,2629)\end{array}$ & 0,09 & $\begin{array}{c}0,5238 \\
(0,2687)\end{array}$ & 0,05 & $\begin{array}{l}1,6851 \\
(0,5050) \\
\end{array}$ & 0,00 \\
\hline$\Delta \ln \left[\bar{Y}_{i t}\right] \ln \left[\frac{z_{i t}}{\bar{y}_{i 0}}\right]$ & $\begin{array}{l}1,064 \\
(0,4832)\end{array}$ & 0,02 & $\begin{array}{l}1,0820 \\
(0,4801)\end{array}$ & 0,05 & $\begin{array}{l}1,1565 \\
(0,3860)\end{array}$ & 0,00 \\
\hline$\Delta \ln \left[\right.$ Gini $\left._{i t}\right]$ & $\begin{array}{c}0,4209 \\
(0,3479)\end{array}$ & 0,22 & $\begin{array}{l}0,4865 \\
(0,3507)\end{array}$ & 0,16 & $\begin{array}{l}3,4064 \\
(0,8328)\end{array}$ & 0,00 \\
\hline$\Delta \ln \left[\operatorname{Gini}_{i t}\right] \ln \left[G_{i 0}\right]$ & $\begin{array}{l}-0,3783 \\
(2,6100) \\
\end{array}$ & 0,53 & $\begin{array}{l}-0,5010 \\
(0,6166)\end{array}$ & 0,41 & $\begin{array}{l}-5,6068 \\
(1,4515)\end{array}$ & 0,00 \\
\hline$\Delta \ln \left[\operatorname{Gini}_{i t}\right] \ln \left[\frac{z_{i t}}{\bar{y}_{i 0}}\right]$ & $\begin{array}{l}-2,998 \\
(0,8709)\end{array}$ & 0,00 & $\begin{array}{l}-3,0703 \\
(0,8771)\end{array}$ & 0,00 & $\begin{array}{l}-1,2865 \\
(0,6120)\end{array}$ & 0,05 \\
\hline $\ln \left[G_{i 0}\right]$ & $\begin{array}{c}0,1283 \\
(0,1121)\end{array}$ & 0,25 & & - & $\begin{array}{l}1,1980 \\
(0,5580)\end{array}$ & 0,04 \\
\hline $\ln \left[\frac{z_{i t}}{\bar{y}_{i 0}}\right]$ & $\begin{array}{l}-0,0931 \\
(0,0377)\end{array}$ & 0,01 & $\begin{array}{l}-0,2159 \\
(0,0479)\end{array}$ & 0,00 & $\begin{array}{c}0,2876 \\
(0,6176)\end{array}$ & 0,00 \\
\hline \multirow[t]{2}{*}{ Constante } & $\begin{array}{c}0,0851 \\
(0,0650)\end{array}$ & 0,19 & $\begin{array}{c}0,0491 \\
(0,1344)\end{array}$ & 0,00 & $\begin{array}{c}0,7001 \\
(0,3002)\end{array}$ & 0,03 \\
\hline & \multicolumn{2}{|c|}{$\begin{array}{l}F(9,63)=21,93 \\
P r o b>F=0,0000 \\
R^{2}=0,43\end{array}$} & \multicolumn{2}{|c|}{$\begin{array}{l}F(8,244)=26,63 \\
\text { Prob }>F=0,0000\end{array}$} & \multicolumn{2}{|c|}{$\begin{array}{l}F(8,20)=16,24 \\
\text { Prob }>F=0,0000\end{array}$} \\
\hline \multicolumn{3}{|c|}{$\begin{array}{l}\mathrm{H}_{0}: \text { : Ausencia de autocorrelación en los } \\
\text { residuales de primer orden }\end{array}$} & \multicolumn{2}{|l|}{ Valor $p$} & \multicolumn{2}{|c|}{0,002} \\
\hline \multicolumn{3}{|c|}{$\begin{array}{l}\mathrm{H}_{0}: \text { : Ausencia de autocorrelación en los } \\
\text { residuales de segundo orden }\end{array}$} & \multicolumn{2}{|l|}{ Valor $p$} & \multicolumn{2}{|c|}{0,829} \\
\hline \multicolumn{3}{|c|}{ Prueba de Hansen } & \multicolumn{2}{|c|}{ Prob $>$ chi $^{2}$} & \multicolumn{2}{|c|}{0,360} \\
\hline \multicolumn{3}{|l|}{ Prueba de Sargan } & \multicolumn{2}{|c|}{ Prob > chi ${ }^{2}$} & \multicolumn{2}{|c|}{0,269} \\
\hline
\end{tabular}

Fuente: Elaboración propia.

Nota: i) Los valores entre paréntesis son las desviaciones estándar corregidas con el método de Windmeijer (2005); ii) los valores de la prueba de Hansen son los valores p de la hipótesis nula de que los instrumentos son válidos; iii) los valores de la prueba de Sargan son los valores $\mathrm{p}$ correspondientes a la validez de los instrumentos adicionales requeridos por el sistema MGM, donde se toman las variables explicativas en diferencias retrasadas como instrumentos para el sistema MGM y se retrasa $\Delta\left[\Delta \ln \left[P_{0, i t-1}\right]\right]$ y $\Delta\left[\Delta \ln \left[\bar{y}_{i t}\right]\right]$ un período, y iv) se dispone de 273 observaciones, 21 grupos y 17 instrumentos.

Como en forma aislada el ingreso medio tiene un efecto negativo en la pobreza, un incremento del ingreso tendrá menor incidencia sobre la lucha contra la pobreza que cuando el nivel de desarrollo inicial es bajo. Lo mismo sucede cuando el índice de desigualdad inicial es alto. Estos resultados confirman conclusiones a las que arribaron Medina y Galván (2014b), que calcularon elasticidades relativas a la pobreza y determinaron cómo se modificaban los indicadores de pobreza, las variaciones en los ingresos y la curva de Lorenz. Utilizaron bases de datos sobre encuestas de hogares de 18 países de América Latina que abarcaron de 1997 a 2000 y el quinquenio 2002-2007. El estudio tuvo como objetivo medir en qué grado el ingreso y la desigualdad pueden afectar la mitigación de la pobreza en escenarios contrafactuales simulados que permiten conocer la sensibilidad de los índices de pobreza a cambios en el ingreso y el nivel de desigualdad. El análisis se llevó a cabo utilizando toda la información sobre la distribución del ingreso disponible en cada país. A partir de la aplicación de métodos de descomposición que permiten separar los cambios en la pobreza en efectos de crecimiento del ingreso y de desigualdad, se simulan escenarios contrafactuales que permiten conocer 
la importancia de los cambios en la inequidad del ingreso a partir de la tasa marginal proporcional de sustitución (TMPS) propuesta por Kakwani. Los resultados parecen indicar que no es correcto sugerir a todos los países las mismas opciones de política, debido a que la sensibilidad de la tasa de pobreza depende de su nivel inicial, así como del grado de desigualdad existente.

En consonancia con ello, se puede afirmar que en regiones con bajos niveles de desarrollo inicial o con una elevada desigualdad inicial, o ambos, las condiciones para mitigar la pobreza por medio de mayores ingresos resultan relativamente desfavorables. Así, podemos concluir que la elevada desigualdad y el escaso nivel de desarrollo inicial de la mayoría de los estados del Brasil constituyen obstáculos para aliviar la pobreza mediante mejores ingresos.

En cuanto al coeficiente estimado en la columna [c] relativo a la interacción entre el cambio en la desigualdad y el nivel de desarrollo inicial inverso, resulta negativo y estadísticamente significativo. Lo mismo ocurre con la interacción entre la variable de desigualdad y su nivel inicial. Así, el efecto de los cambios en la desigualdad en la reducción de la pobreza es más acotado cuando el nivel de desarrollo inicial es bajo o cuando la desigualdad inicial presenta un nivel elevado. En otras palabras, una disminución en la desigualdad de los ingresos puede resultar menos efectiva para reducir la pobreza en regiones que adolecen de un desarrollo inicial bajo o de una alta desigualdad inicial, o de ambos.

Por lo tanto, en el Brasil el bajo nivel de desarrollo inicial y la alta desigualdad inicial de ingreso dificultan la reducción de la pobreza, independientemente de si esta meta va acompañada de esfuerzos para estimular el crecimiento económico o reducir la desigualdad de los ingresos.

Este hecho se ve corroborado por los hallazgos de Medina y Galván (2014a), que emplearon diferentes metodologías econométricas para analizar en qué grado el crecimiento económico y la desigualdad contribuyen a la evolución de la pobreza mediante una base de datos de encuestas de hogares llevadas a cabo en 18 países de América Latina. El progreso logrado respecto de la desigualdad -y un aumento del ingreso per cápita- explican el declive en el nivel de pobreza experimentado en el período 2002-2007. Los resultados confirman que es posible reducir la pobreza a partir de políticas que tengan como objetivo prioritario reducir el nivel de inequidad en la distribución del ingreso, sobre todo en los países de mayor desarrollo, en tanto que en las economías de menores recursos se requiere, además, incrementar el ingreso de las familias menos favorecidas como condición necesaria para reducir la pobreza. Estos autores hallaron que una combinación adecuada de políticas que incrementen el ingreso y mejoren su distribución generaría un círculo virtuoso de reducción rápida y sostenida de la pobreza. A todas luces, la sensibilidad de los indicadores de pobreza a los cambios del ingreso familiar medio se correlaciona con el nivel de desigualdad, por lo que, si se disminuye la desigualdad de los ingresos, se mejora la reducción de la pobreza.

El coeficiente de desarrollo inicial inverso tiene una relación positiva y estadísticamente significativa con el porcentaje de pobres (valores de la columna [c]). Por ello, cuanto mayor sea el nivel de desarrollo inicial inverso, mayor será la incidencia de la pobreza. O dicho con otras palabras: a menor ingreso familiar inicial per cápita, mayor incidencia de la pobreza.

Las últimas filas del cuadro 4 presentan los resultados de la prueba de Arellano y Bond (1991) para las autocorrelaciones residuales de primer y segundo orden, y las pruebas de Hansen y Sargan orientadas a determinar la validez de los instrumentos. Si se toman los valores $p$ de la columna [c], los resultados de las pruebas de Arellano y Bond indican que se puede refutar la hipótesis nula de ausencia de autocorrelación de primer orden y aceptar la existencia de una autocorrelación residual de segundo orden. Los valores $p$ de las pruebas de Hansen y Sargan nos permiten aceptar la hipótesis de que los instrumentos usados en las estimaciones del modelo son válidos. 


\section{Elasticidades pobreza-ingreso y pobreza-desigualdad en los estados del Brasil}

Las elasticidades pobreza-ingreso y pobreza-desigualdad correspondientes a los estados brasileños se calcularon utilizando las fórmulas (10) y (11). Los parámetros de estas dos expresiones se obtuvieron a partir de la estimación del segundo modelo, pues resultaba más adecuado para determinar estas elasticidades, dado que tomaba en cuenta las características de la distribución del ingreso, el nivel de desigualdad y el nivel de desarrollo inicial. En el cuadro 5 se presentan las elasticidades medias de las regiones y los estados del Brasil de 1995 a 2009, así como las desviaciones estándar estimadas.

\section{Cuadro 5}

Brasil (estados y regiones): elasticidades medias pobreza-ingreso y pobreza-desigualdad

\begin{tabular}{|c|c|c|c|c|}
\hline Estado & $\begin{array}{c}\text { Elasticidad } \\
\text { pobreza-ingreso }\end{array}$ & $\begin{array}{l}\text { Desviación } \\
\text { estándar }\end{array}$ & $\begin{array}{c}\text { Elasticidad } \\
\text { pobreza-desigualdad }\end{array}$ & $\begin{array}{l}\text { Desviación } \\
\text { estándar }\end{array}$ \\
\hline Maranhão & $-1,61$ & & 2,33 & \\
\hline Piauí & $-1,52$ & & 2,30 & \\
\hline Ceará & $-1,50$ & & 2,34 & \\
\hline Rio Grande do Norte & $-1,54$ & & 2,30 & \\
\hline Paraíba & $-1,53$ & & 2,40 & \\
\hline Pernambuco & $-1,56$ & & 2,52 & \\
\hline Alagoas & $-1,55$ & & 2,50 & \\
\hline Sergipe & $-1,56$ & & 2,47 & \\
\hline Bahia & $-1,57$ & & 2,40 & \\
\hline Nordeste & $-1,54$ & 0,03 & 2,39 & 0,08 \\
\hline Minas Gerais & $-1,58$ & & 2,48 & \\
\hline Espírito Santo & $-1,61$ & & 2,51 & \\
\hline Río de Janeiro & $-1,63$ & & 2,49 & \\
\hline São Paulo & $-1,61$ & & 2,42 & \\
\hline Sudeste & $-1,60$ & 0,02 & 2,47 & 0,03 \\
\hline Paraná & $-1,59$ & & 2,49 & \\
\hline Santa Catarina & $-1,65$ & & 2,38 & \\
\hline Rio Grande do Sul & $-1,64$ & & 2,47 & \\
\hline Sur & $-1,62$ & 0,03 & 2,44 & 0,05 \\
\hline Mato Grosso do Sul & $-1,58$ & & 2,49 & \\
\hline Mato Grosso & $-1,61$ & & 2,49 & \\
\hline Goiás & $-1,59$ & & 2,51 & \\
\hline Distrito Federal & $-1,62$ & & 2,50 & \\
\hline Centro-Oeste & $-1,60$ & 0,01 & 2,49 & 0,009 \\
\hline
\end{tabular}

Fuente: Elaboración propia.

Como cabe esperar a partir de las señales de las elasticidades teóricas pobreza-ingreso y pobrezadesigualdad presentadas en la sección III, la primera es negativa en todos los estados y regiones del Brasil, mientras que la segunda es positiva. En otras palabras, el crecimiento del ingreso medio y la reducción de la desigualdad de los ingresos redundaron en una merma del número de pobres.

No obstante, como se muestra en el cuadro 5, los valores de estas elasticidades revelan que los cambios en la desigualdad de los ingresos ejercieron en la lucha contra la pobreza un efecto más profundo que el aumento del ingreso medio. Esto se condice con los hallazgos de Kakwani (1990) y de Marinho y Soares (2003).

En cuanto al plano regional, los valores absolutos de la elasticidad pobreza-ingreso resultan ser más bajos en el nordeste que en las demás regiones. Este resultado confirma la hipótesis teórica de que la elasticidad pobreza-ingreso es menor en economías con un ingreso medio menos cuantioso. 
En las regiones más ricas, el aumento del ingreso medio tiene repercusiones más profundas en la reducción de la pobreza. Estos resultados corroboran las conclusiones extraídas por Marinho y Soares (2003) y por Hoffmann (2004). Por consiguiente, a las regiones menos desarrolladas, como el nordeste del Brasil, les resulta más difícil reducir la pobreza mediante la mejora de los ingresos. De manera similar, la elasticidad pobreza-desigualdad también es menor en el nordeste que en las restantes regiones, pero los cambios en el nivel de desigualdad tienen un efecto en la pobreza mayor que el aumento del ingreso medio.

En términos generales, estos resultados parecen indicar que las políticas orientadas a reducir la desigualdad son la herramienta más útil para luchar contra la pobreza en el Brasil.

\section{Conclusiones}

Este artículo tiene como objeto estimar las elasticidades de la pobreza en función del ingreso y la desigualdad en el Brasil con miras a analizar los determinantes de la reducción de la pobreza. Más específicamente, se evalúa si los cambios en el nivel de pobreza son producto de la redistribución del ingreso, del crecimiento económico o de ambo, y se examina la influencia que cada uno de estos efectos ejerce en las variaciones del nivel de pobreza.

Los resultados de la estimación del primer modelo mostraron que las elasticidades pobrezaingreso y pobreza-desigualdad eran de $-0,68$ y 0,77 , respectivamente. Ello entraña que un aumento del $1,0 \%$ en el ingreso per cápita redunda en una merma del 0,68\% en el porcentaje de personas pobres. De igual modo, un aumento de la desigualdad de un 1,0\% conlleva un crecimiento de la pobreza del $0,77 \%$. Cabe recordar que estos resultados corroboran hallazgos de estudios internacionales, como los de Kalwij y Verschoor (2004), y Bourguignon (2004), así como de estudios llevados a cabo en el Brasil, como los de Marinho y Soares (2003) y Hoffmann (2004). La implicancia de estos hallazgos es que las políticas orientadas a reducir la desigualdad son más efectivas para reducir la pobreza que aquellas solamente destinadas a mejorar el ingreso medio.

Los resultados estimados para el segundo modelo, que permite que las elasticidades varíen con el tiempo, mostraron que los factores que contribuían al aumento de la pobreza eran, en orden de importancia ascendente: la interacción entre los cambios en los ingresos y el nivel de desarrollo inicial inverso, la desigualdad inicial de los ingresos, la interacción entre los cambios en los ingresos y la desigualdad inicial de los ingresos, y la desigualdad de los ingresos en la actualidad.

El grado en que el crecimiento de los ingresos repercute en la reducción de la pobreza es más acotado cuando el nivel de desarrollo inicial es bajo y cuando la desigualdad inicial es alta. Por ende, podemos concluir que las regiones con un nivel de desarrollo inicial bajo o una desigualdad inicial alta - o en presencia de ambos factores - se encuentran en una posición menos favorable para reducir la pobreza mediante la mejora de los ingresos. En consecuencia, resulta evidente que la marcada desigualdad y el exiguo desarrollo inicial de la mayoría de los estados brasileños son un escollo para reducir la pobreza incrementando los ingresos.

De igual modo, el efecto que los cambios en la desigualdad ejercen en la reducción la pobreza es más acotado cuando el nivel de desarrollo inicial es bajo o cuando la desigualdad inicial presenta un nivel elevado. Así, el intento por combatir la pobreza reduciendo la desigualdad de ingresos en las regiones o estados brasileños que padecen un bajo nivel de desarrollo inicial, una alta desigualdad inicial, o ambos, puede no tener el resultado esperado.

Como ya se señaló, el limitado desarrollo inicial y la marcada desigualdad inicial presentes en el Brasil dificultan la reducción de la pobreza, independientemente de que la consecución de este objetivo se base en estimular el crecimiento económico o en atenuar la desigualdad de los ingresos. 
Respecto de las elasticidades pobreza-ingreso y pobreza-desigualdad, se determinó que la desigualdad de los ingresos tiene sobre la pobreza un impacto más profundo que el aumento del ingreso medio. Este efecto también fue observado por Kakwani (1990) y por Marinho y Soares (2003).

En el plano regional, el valor absoluto de la elasticidad pobreza-ingreso es menor en el nordeste que en las demás regiones del Brasil. Este resultado corrobora la hipótesis teórica de que la elasticidad pobreza-ingreso es menor en economías con un ingreso medio bajo. En regiones más ricas, el incremento del ingreso medio tiene un efecto más potente en la reducción de la pobreza. Los resultados obtenidos en este análisis se condicen con los hallazgos de Marinho y Soares (2003) y de Hoffmann (2004). En síntesis, a las regiones menos desarrolladas, como el nordeste del Brasil, les resulta más difícil reducir la pobreza mediante la mejora de los ingresos.

De igual modo, la elasticidad pobreza-desigualdad es menor en el nordeste que en las otras regiones del país, pero la influencia que la desigualdad ejerce en la pobreza es más fuerte que la del aumento del ingreso medio. En líneas generales, estos resultados indican que, para erradicar la pobreza en el Brasil, las políticas más exitosas serán aquellas que apunten a reducir la desigualdad.

\section{Bibliografía}

Ahn, S. C. y P. Schmidt (1995), "Efficient estimation of models for dynamic panel data", Journal of Econometrics, vol. 68, No 1, Amsterdam, Elsevier.

Alesina, A. y D. Rodrik (1994), "Distributive policies and economic growth", Quarterly Journal of Economics, vol. 109, № 2, Oxford University Press.

Arellano, M. y O. Bover (1995), "Another look at the instrumental variable estimation of error-components model", Journal of Econometrics, vol. 68, № 1, Amsterdam, Elsevier.

Arellano, M. y S. Bond (1991), "Some tests of specification for panel data: Monte Carlo evidence and an application to employment equations", The Review of Economic Studies, vol. 58, №2, Oxford University Press.

Barreto, F. A. F. D. (2005), “Crescimento econômico, pobreza e desigualdade: o que sabemos sobre eles?”, Série Ensaios sobre Pobreza, № 1, Fortaleza, Universidad Federal de Ceará.

Barro, R. (2000), "Inequality and growth in a panel of countries", Journal of Economic Growth, vol. 5, № 1.

Barros, R. P. de y otros (2007), "Determinantes da queda da desigualdade de renda Brasileira", Texto para Discussão, № 1253, Río de Janeiro, Instituto de Investigación Económica Aplicada (IPEA).

Barros, R. P. de, R. Henriques y R. Mendonça (2001), "A estabilidade inaceitável: desigualdade e pobreza no Brasil”, Texto para Discussão, № 800, Río de Janeiro, Instituto de Investigación Económica Aplicada (IPEA).

Blundell, R. y S. Bond (1998), "Initial conditions and moment restrictions in dynamic panel data models", Journal of Econometrics, vol. 87, № 1, Amsterdam, Elsevier.

Bourguignon, F. (2004), "The Poverty-Growth-Inequality Triangle”, Washington, D.C., Banco Mundial [en línea] http://siteresources.worldbank.org/INTPGI/Resources/342674-1206111890151/15185_ICRIER_paperfinal.pdf.

(2003), "The growth elasticity of poverty reduction: explaining heterogeneity across countries and time periods", Inequality and Growth: Theory and Policy Implications, T. Eicher y S. Turnovsky (eds.), Cambridge, Massachusetts, The MIT Press.

(1981), "Pareto superiority of unegalitarian equilibria in Stiglitz' model of wealth distribution with convex saving function", Econometrica, vol. 49, № 6, Nueva York, Econometric Society.

Chen, S. e Y. Wang (2001), "China's growth and poverty reduction: recent trends between 1990 and 1999", Policy Research Working Paper, № 2651, Washington, D.C., World Bank.

Cline, W. R. (2004), Trade Policy and Global Poverty, Washington, D.C., Center for Global Development/ Instituto de Economía Internacional.

Datt, G. y M. Ravallion (2002), "Is India's economic growth leaving the poor behind?", Journal of Economic Perspectives, vol. 16, № 3, Nashville, Tennessee, American Economic Association.

Deininger, K. y L. Squire (1996), "A new data set measuring income inequality", World Bank Economic Review, vol. 10, №3, Washington, D.C., Banco Mundial. 
Epaulard, A. (2003), "Macroeconomic performance and poverty reduction", IMF Working Paper, № 03/72, Washington, D.C., Fondo Monetario Internacional (FMI).

Foster, J., J. Greer y E. Thorbecke (1984), "A class of decomposable poverty measures", Econometrica, vol. 52, № 3, Nueva York, Econometric Society.

Hoffmann, R. (2007), "Transferência de renda e a redução da desigualdade no Brasil e em cinco regiões entre 1997 e 2005", Desigualdade de renda no Brasil: uma análise da queda recente, vol. 2, R. Paes de Barros, M. N. Foguel y G. Ulyssea (orgs.), Brasilia, Instituto de Investigación Económica Aplicada (IPEA). (2005), "Elasticidade da pobreza em relação à renda média e à desigualdade no Brasil e nas unidades da federação", Economia, vol. 6, Nㅜㄹ, Asociación Nacional de Centros de Postgrado en Economía (ANPEC). (2004), "Elasticidade da pobreza em relação à renda média e à desigualdade" [en línea] http://www. anpec.org.br/encontro2004/artigos/A04A054.pdf.

_ (1995), "Desigualdade e pobreza no Brasil no período 1970-1990, Revista Brasileira de Economia, vol. 49, No2.

Kakwani, N. (1990), "Poverty and economic growth: with application to Côte d'Ivoire", Living Standards Measurement Study Working Paper, № 63, Washington, D.C., Banco Mundial.

Kalwij, A. y A. Verschoor (2004), "How good is growth for the poor? The role of the initial income distribution in regional diversity in poverty trends", CentER Discussion Paper Series, № 115, CentER-Universidad de Tillburg.

Kraay, A. (2004), "When is growth pro-poor? Evidence from a panel of countries", Washington, D.C., Policy Research Working Paper, Nº 3225, Washington, D.C., Banco Mundial.

Kuznets, S. (1955), "Economic growth and income inequality", American Economic Review, vol. 45, № 1, Nashville, Tennessee, American Economic Association.

Judson, R. A. y A. L. Owen (1999), "Estimating dynamic panel data models: a guide for macroeconomists", Economics Letters, vol. 65, № 1, Amsterdam, Elsevier.

López, J. H. (2004), "Pro-growth, pro-poor: is there a trade-off?", Policy Research Working Paper Series, $N^{\circ} 3378$, Washington, D.C., Banco Mundial.

Manso, C. A., F. A. Barreto y E. Tebaldi (2005), "O desequilíbrio regional brasileiro: novas perspectivas a partir das fontes de crescimento pró-pobre", Série Ensaios sobre Pobreza, № 6, Fortaleza, Universidad Federal de Ceará.

Marinho, E. y F. Soares (2003), "Impacto do crescimento econômico e da concentração de renda sobre a redução da pobreza nos estados brasileiros" [en línea] http://www.anpec.org.br/encontro2003/artigos/ B36.pdf.

Medina, F. y M. Galván (2014a), "Crecimiento económico, pobreza y distribución del ingreso. Fundamentos teóricos y evidencia empírica para América Latina, 1997-2007", serie Estudios Estadísticos, № 82 (LC/L.3689), Santiago, Comisión Económica para América Latina y el Caribe (CEPAL).

(2014b), "Sensibilidad de los índices de pobreza a los cambios en el ingreso y la desigualdad. Lecciones para el diseño de políticas en América Latina, 1997-2008", serie Estudios Estadísticos, № 87 (LC/L.3823), Santiago, Comisión Económica para América Latina y el Caribe (CEPAL).

Neri, M. (2006), "Desigualdade, estabilidade e bem-estar social", Ensaios Econômicos, № 637, Río de Janeiro, Fundación Getulio Vargas.

Ravallion, M. (2005), "Pro-poor growth: a primer", Policy Research Working Paper, № 3242, Washington, D.C., Banco Mundial.

(2001), "Growth, inequality and poverty: looking beyond averages", World Development, vol. 29, № 11, Amsterdam, Elsevier.

Ravallion, M. y S. Chen (1997), "What can new survey data tell us about recent changes in distribution and poverty?", World Bank Economic Review, vol. 11, № 2, Washington, D.C., Banco Mundial.

Ribas, R. P., A. F. Machado y A. B. Golgher (2006), "Fluctuations and persistence in poverty: a transientchronic decomposition model for pseudo-panel data", Texto para Discussão, Nㅜ290, Universidad Federal de Minas Gerais.

Rocha, S. W. W. (2006), Pobreza no Brasil. Afinal, de que se trata?, Río de Janeiro, FGV Editora.

Salvato, M. A. y A. F. de Araujo Junior (2007), "Crescimento pró-pobre no Brasil: uma avaliação empírica da década de 1990", Texto para Discussão, № 02, Minas Gerais, Pontificia Universidad Católica de Minas Gerais.

Stewart, F. (2000), Distribuição de renda e desenvolvimento, Brasilia, NEAD.

Son, H. H. (2004), "A note on pro-poor growth", Economics Letters, vol. 82, № 3, Amsterdam, Elsevier.

Windmeijer, F. (2005), "A finite sample correction for the variance of linear efficient two-step GMM estimators", Journal of Econometrics, vol. 126, № 1, Amsterdam, Elsevier. 